\title{
ON THE ASYMPTOTIC BEHAVIOR OF SOLUTIONS OF CERTAIN THIRD-ORDER NONLINEAR DIFFERENTIAL EQUATIONS
}

\author{
CEMIL TUNÇ
}

Received 25 April 2004 and in revised form 15 July 2004

We establish sufficient conditions under which all solutions of the third-order nonlinear differential equation $\dddot{x}+\psi(x, \dot{x}, \ddot{x}) \ddot{x}+f(x, \dot{x})=p(t, x, \dot{x}, \ddot{x})$ are bounded and converge to zero as $t \rightarrow \infty$.

\section{Introduction}

The differential equation considered here is of the form

$$
\dddot{x}+\psi(x, \dot{x}, \ddot{x}) \ddot{x}+f(x, \dot{x})=p(t, x, \dot{x}, \ddot{x}),
$$

where $\psi \in C(\mathbb{R} \times \mathbb{R} \times \mathbb{R}, \mathbb{R}), f \in C(\mathbb{R} \times \mathbb{R}, \mathbb{R})$, and $p \in C([0, \infty) \times \mathbb{R} \times \mathbb{R} \times \mathbb{R}, \mathbb{R})$. It is also supposed that the functions $\psi, f$, and $p$ depend only on the arguments displayed explicitly, and the dots denote differentiation with respect to $t$. However, we shall require that $f(0,0)=0$, the derivatives $\partial \psi(x, \dot{x}, \ddot{x}) / \partial x \equiv \psi_{x}(x, \dot{x}, \ddot{x}), \partial \psi(x, \dot{x}, \ddot{x}) / \partial \ddot{x} \equiv \psi_{\ddot{x}}(x, \dot{x}, \ddot{x})$, and $\partial f(x, \dot{x}) / \partial x \equiv f_{x}(x, \dot{x})$ exist and are continuous, and the uniqueness of the solutions of (1.1) will be assumed.

In relevant literature, a good deal of work has been done and many interesting results have been obtained concerning the asymptotic behavior of solutions in the particular cases of (1.1), see, for example, $[1,2,3,4,5,6,7,8,9,10,11,12,13,14,15,16]$ and the references cited therein. In the relevant references, the authors dealt with the problems and obtained the criteria for the asymptotic behavior of the solutions by employing Lyapunov functions as a main tool. In this matter, Singh [13] discussed the asymptotic behavior of solutions of the third-order linear differential equations of the form

$$
\ddot{y}+p(t) \dot{y}+q(t) y=0 .
$$

In [2], Ezeilo investigated the stability of solutions of the differential equation

$$
\dddot{x}+\psi(x, \dot{x}) \ddot{x}+\phi(\dot{x})+g(x)=p(t, x, \dot{x}, \ddot{x}) .
$$


Swick [14] studied the asymptotic behavior of solutions of the nonlinear differential equations

$$
\begin{gathered}
\dddot{x}+a \ddot{x}+g(x) \dot{x}+h(x)=e(t), \\
\dddot{x}+p(t) \ddot{x}+q(t) g(\dot{x})+h(x)=e(t),
\end{gathered}
$$

respectively.

In [5], Nakashima considered the differential equations

$$
\begin{gathered}
\dddot{x}+a \ddot{x}+g(x) \dot{x}+h(x)=e(t, x, \dot{x}, \ddot{x}), \\
\dddot{x}+p(t) \ddot{x}+q(t) g(\dot{x})+h(x)=e(t, x, \dot{x}, \ddot{x}),
\end{gathered}
$$

and established some results on the qualitative behavior of solutions of the equations. Goldwyn and Narendra [3] also studied the same subject for the following third-order nonlinear differential equation:

$$
\dddot{x}+h(\dot{x}) \ddot{x}+\mu(\dot{x}) \dot{x}+k(x) x=0 .
$$

Recently, Qian [7, 8] discussed the global stability and asymptotic behavior of solutions of the equations of the form

$$
\begin{aligned}
& \dddot{x}+\psi(x, \dot{x}) \ddot{x}+f(x, \dot{x})=0, \\
& \dddot{x}+\psi(x, \dot{x}) \ddot{x}+f(x, \dot{x})=p(t),
\end{aligned}
$$

respectively.

The motivation for the present work has come from the papers mentioned above. Our aim is to obtain two similar results for (1.1). Namely, we will present here sufficient conditions, which ensure that all solutions of (1.1) are uniformly bounded and converge to zero as $t \rightarrow \infty$.

In what follows we use the following differential system which is equivalent to (1.1):

$$
\dot{x}=y, \quad \dot{y}=z, \quad \dot{z}=-\psi(x, y, z) z-f(x, y)+p(t, x, y, z) .
$$

\section{Boundedness of solutions}

In this section, the following result is established. For the proof of the result Lyapunov's second method is utilized.

Theorem 2.1. Further to the basic assumptions on the functions $\psi, f$, and $p$ suppose the following:

(i) $\int_{0}^{x} f(u, 0) d u>0$ for $x \neq 0$;

(ii) $\lim _{|x| \rightarrow \infty} \sup \int_{0}^{x} f(u, 0) d u=\infty$;

(iii) $\int_{0}^{y} f(0, v) d v \geq 0$;

(iv) the function $p$ satisfies $|p(t, x, y, z)| \leq|e(t)|$ uniformly in $t$, where $e(t)$ is a continuous function of $t$ such that $\int_{0}^{\infty}|e(t)| d t<\infty$;

(v) there is a positive constant B such that

$$
\psi(x, y, z) \geq B
$$


(vi) $B\left[f(x, y)-f(x, 0)-\int_{0}^{y} \psi_{x}(x, v, 0) v d v\right] y \geq y \int_{0}^{y} f_{x}(x, v) d v$;

(vii) $4 B \int_{0}^{x} f(u, 0) d u\left\{\int_{0}^{y}[f(x, v)-f(x, 0)] d v+B \int_{0}^{y}[\psi(x, v, 0)-B] v d v\right\} \geq y^{2} f^{2}(x, 0)$ for all $x y \neq 0$;

(viii) $y \psi_{z}(x, y, z) \geq 0$.

Then for any solution $(x(t), y(t), z(t))$ of system (1.8), there are positive constants $c_{1}, c_{2}$, and $c_{3}$ such that

$$
|x(t)|<c_{1}, \quad|y(t)|<c_{2}, \quad|z(t)|<c_{3} \quad \text { for } t \geq 0 .
$$

Remark 2.2. The theorem just stated above improves the theorem established in [1] and includes the result established in [8, Theorem 1]. The results of Ezeilo [2], Ogurtsov [6], and Goldwyn and Narendra [3] are also direct consequences of our result.

Proof of Theorem 2.1. The proof depends on a scalar differentiable comparison Lyapunov function $V=V(t, x, y, z)$. This function and its total time derivative satisfy some fundamental inequalities. We define $V$ as follows:

$$
V=e^{-P(t)}\left[B F(x)+y f(x, 0)+\Phi(x, y)+B \int_{0}^{y}[\psi(x, v, 0)-B] v d v+\frac{(z+B y)^{2}}{2}+2\right] \text {, }
$$

where

$$
P(t)=\int_{0}^{t}|e(s)| d s, \quad F(x)=\int_{0}^{x} f(u, 0) d u, \quad \Phi(x, y)=\int_{0}^{y}[f(x, v)-f(x, 0)] d v .
$$

First we show that $V$ is a positive-definite function. For the sake of convenience, let

$$
G(x, y)=B F(x)+y f(x, 0)+\Phi(x, y)+B \int_{0}^{y}[\psi(x, v, 0)-B] v d v .
$$

Then $V$ can be written as follows:

$$
V(t, x, y, z)=e^{-P(t)}\left[G(x, y)+\frac{(z+B y)^{2}}{2}+2\right] .
$$

Now, it is clear from (2.6) that if $G(x, y)$ is nonnegative, then $V$ is a positive-definite function. Indeed, observe that when $x=0$,

$$
G(0, y)=\Phi(0, y)+B \int_{0}^{y}[\psi(0, v, 0)-B] v d v
$$

Hence, in view of the assumptions (iii), (v) of Theorem 2.1 and $f(0,0)=0$, it is easy to see that $G(0, y) \geq 0$. Next, assume that $x \neq 0$. Then $G(x, y)$ can be rearranged as

$$
G(x, y)=\left[\sqrt{B F(x)}+\frac{1}{2} \frac{y f(x, 0)}{\sqrt{B F(x)}}\right]^{2}+\left[\Phi(x, y)+B \int_{0}^{y}(\psi(x, v, 0)-B) v d v-\frac{y^{2} f^{2}(x, 0)}{4 b F(x)}\right] .
$$


The assumption (vii) of Theorem 2.1 also shows that $G(x, y) \geq 0$. So, it can be followed that $V$ is a positive-definite function.

By a straightforward elementary differentiation from (2.3) and (1.8) it can be verified that

$$
\begin{aligned}
\frac{d V}{d t}=\dot{V}= & \left\{-\left|e^{-P(t)}\right|\left[G(x, y)+\frac{(z+B y)^{2}}{2}+2\right]\right\} \\
& +e^{-P(t)} y\left\{B\left[f(x, 0)-f(x, y)+\int_{0}^{y} \psi_{x}(x, v, 0) v d v\right]+\int_{0}^{y} f_{x}(x, v) d v\right\} \\
& +e^{-P(t)}[B-\psi(x, y, z)] z^{2}+e^{-P(t)}(z+B y) p(t, x, y, z)-e^{-P(t)} W,
\end{aligned}
$$

where

$$
W \equiv B \psi(x, y, z) y z-B \psi(x, y, 0) y z
$$

Next, we show that $d V / d t=\dot{V} \leq 0$.

By noting the assumptions (v), (viii) of Theorem 2.1 and the mean value theorem for derivative, it follows that

$$
W=B y z^{2}\left[\frac{\psi(x, y, z)-\psi(x, y, 0)}{z}\right]=B y z^{2} \psi_{z}(x, y, \theta z) \geq 0, \quad 0 \leq \theta \leq 1
$$

but $W=0$ when $z=0$. Hence

$$
W \geq 0 \text {. }
$$

In addition, observe that the assumption (iv) of Theorem 2.1 implies that $|z+B y|<2$, then

$$
(z+B y) p(t, x, y, z) \leq 2|p(t, x, y, z)| \leq 2|e(t)|
$$

if $|z+B y| \geq 2$, then

$$
(z+B y) p(t, x, y, z) \leq \frac{(z+B y)^{2}}{2}|p(t, x, y, z)| \leq \frac{(z+B y)^{2}}{2}|e(t)| .
$$

Hence, for any $t, x, y$, and $z$, we get

$$
(z+B y) p(t, x, y, z) \leq\left(2+\frac{(z+B y)^{2}}{2}\right)|p(t, x, y, z)| \leq\left(2+\frac{(z+B y)^{2}}{2}\right)|e(t)| .
$$

On gathering the estimates (2.12) and (2.15) into (2.9) we obtain

$$
\begin{aligned}
\dot{V} \leq & e^{-P(t)}\left\{-|e(t)| G(x, y)+[B-\psi(x, y, z)] z^{2}\right\} \\
& +e^{-P(t)} y\left\{B\left[f(x, 0)-f(x, y)+\int_{0}^{y} \psi_{x}(x, v, 0) v d v\right]+\int_{0}^{y} f_{x}(x, v) d v\right\} .
\end{aligned}
$$


In view of the assumptions (v), (vi) of Theorem 2.1 and $G(x, y) \geq 0$, it is obvious that $\dot{V} \leq 0$. Finally, we show that all solutions of (1.8) are bounded. Assume that $(x(t), y(t)$, $z(t))$ is a solution of $(1.8)$ with the initial condition

$$
(x(0), y(0), z(0))=\left(x_{0}, y_{0}, z_{0}\right) .
$$

The remaining proof of Theorem 2.1 is similar to that of Qian [8, Theorem 1] and hence it is omitted.

\section{Asymptotic behavior of solutions}

The following lemma is important for the proof of our next theorem.

Lemma 3.1. Let $Q$ be an open set in $\mathbb{R}^{n}$ and $I=[0, \infty)$. Consider the differential system

$$
\frac{d x}{d t}=H(x)+G(t, x)
$$

where $H$ is continuous on $Q, G$ is continuous on $I \times Q$, and for any continuous and bounded function $x(t)$ on $t_{0} \leq t<\infty$,

$$
\int_{0}^{\infty}|G(s, x(s))| d s<\infty .
$$

Assume that all the solutions of (3.1) are bounded, and that there exists a nonnegative continuous function $V(t, x)$ which satisfies locally a Lipschitz condition with respect to $x$ in $Q$ such that $\dot{V}(t, x) \leq-W(x)$, where $W(x)$ is positive definite with respect to a closed set $\Omega$ in $Q$. Then all the solutions of (3.1) approach the largest semi-invariant set contained in $\Omega$ of the equation

$$
\frac{d x}{d t}=H(x)
$$

on $\Omega$.

For the proof of Lemma 3.1, see Yoshizawa [16].

In this section, the following theorem is the main result.

Theorem 3.2. Suppose the following:

(i) there is a positive constant B such that the assumptions (iv)-(viii) of Theorem 2.1 hold;

(ii) $x f(x, 0)>0$ for $x \neq 0$;

(iii) $\lim _{|x| \rightarrow \infty} \sup \int_{0}^{x} f(u, 0) d u=\infty$;

(iv) $\int_{0}^{y} f(0, v) d v \geq 0$;

(v) $B\left[f(x, y)-f(x, 0)-\int_{0}^{y} \psi_{x}(x, v, 0) v d v\right] y+\psi(x, y, z) \geq y \int_{0}^{y} f_{x}(x, v) d v+B$ for $(y \neq 0)$. Then every solution $(x(t), y(t), z(t))$ of system (1.8) satisfies

$$
\lim _{t \rightarrow \infty} x(t)=0, \quad \lim _{t \rightarrow \infty} y(t)=0, \quad \lim _{t \rightarrow \infty} z(t)=0 .
$$

Remark 3.3. Theorem 3.2 improves the result of Ezeilo [2] and generalizes the second result obtained in $[8$, Theorem 2]. 
It should be noted that for the proof of this theorem our main tool is the Lyapunov function $V$ defined by (2.3) again.

Proof of Theorem 3.2. Now, consider the Lyapunov function defined by (2.3) and the system (1.8). As is shown in the proof of Theorem 2.1, because of $G(x, y) \geq 0$, it follows from (2.16) that

$$
\begin{aligned}
\dot{V} \leq & e^{-P(\infty)}[B-\psi(x, y, z)] z^{2} \\
& +e^{-P(\infty)} y\left\{B\left[f(x, 0)-f(x, y)+\int_{0}^{y} \psi_{x}(x, v, 0) v d v\right]+\int_{0}^{y} f_{x}(x, v) d v\right\} .
\end{aligned}
$$

Set

$$
\begin{aligned}
W_{1}(x, y, z)= & -e^{-P(\infty)}[B-\psi(x, y, z)] z^{2} \\
& -e^{-P(\infty)} y\left\{B\left[f(x, 0)-f(x, y)-\int_{0}^{y} \psi_{x}(x, v, 0) v d v\right]+\int_{0}^{y} f_{x}(x, v) d v\right\} .
\end{aligned}
$$

By noting assumptions of the theorem, we see that $W_{1}(x, y, z) \geq 0$. Now, we consider the set

$$
\Omega=\left\{(x, y, z): W_{1}(x, y, z)=0\right\} .
$$

Because the function $W_{1}$ is continuous, the set $\Omega$ is closed and $W_{1}$ is positive definite with respect to $\Omega$. Now, consider the system

$$
\dot{x}=y, \quad \dot{y}=z, \quad \dot{z}=-\psi(x, y, z) z-f(x, y) .
$$

In view of the arguments arising in Qian [8, Theorem 2], it can easily be followed that $(0,0,0)$ is the largest semi-invariant set of (3.8) contained in $\Omega$. Next, because all the hypotheses of Theorem 2.1 are satisfied, it is obvious that every solution of (1.8) is bounded. Now, let

$$
x=(x, y, z)^{T}, \quad H(x)=(x, y,-f(x, y)-\psi(x, y, z) z)^{T}, \quad G(t, x)=(0,0, p(t, x, y, z))^{T} .
$$

Clearly, the system (1.8) is of the form (3.1). Then, from the above discussion, it is easy to check that all the hypotheses in Lemma 3.1 are satisfied. Hence, by Lemma 3.1, every solution of (1.8) tends to the largest semi-invariant set contained in $\Omega$ of (3.8) on $\Omega$, that is, $(0,0,0)$.

It follows thus the original statement of the theorem.

\section{References}

[1] E.A. Barbashin, Lyapunov Functions, Izdat. Nauka, Moscow, 1970.

[2] J. O. C. Ezeilo, On the stability of solutions of certain differential equations of the third order, Quart. J. Math. Oxford Ser. (2) 11 (1960), 64-69.

[3] M. Goldwyn and S. Narendra, Stability of Certain Nonlinear Differential Equations Using the Second Method of Lyapunov, Craft. Lab. Harvard. Univ., Massachusetts, 1963. 
[4] N. N. Krasovski1, Stability of Motion. Applications of Lyapunov's Second Method to Differential Systems and Equations with Delay, Stanford University Press, California, 1963, translated by J. L. Brenner.

[5] M. Nakashima, Asymptotic behavior of the solutions of some third order differential equations, Rep. Fac. Sci. Kagoshima Univ. (1971), no. 4, 7-15.

[6] A. I. Ogurcov, On the stability of the solutions of certain non-linear differential equations of third and fourth order, Izv. Vysš. Učebn. Zaved. Matematika 1959 (1959), no. 3 (10), 200-209.

[7] C. Qian, On global stability of third-order nonlinear differential equations, Nonlinear Anal. Ser. A: Theory Methods 42 (2000), no. 4, 651-661.

[8] - Asymptotic behavior of a third-order nonlinear differential equation, J. Math. Anal. Appl. 284 (2003), no. 1, 191-205.

[9] Y. X. Qin, M. Q. Wang, and L. Wang, Theory and Applications of Stability of Motion, Series of Monographs in Pure and Applied Mathematics, vol. 8, Science Press, Beijing, 1981.

[10] R. Reissig, G. Sansone, and R. Conti, Nonlinear Differential Equations of Higher Order, Noordhoff, Groningen, 1974.

[11] J. D. Schuur, The asymptotic behavior of a solution of the third order linear differential equation, Proc. Amer. Math. Soc. 18 (1967), 391-393.

[12] S. N. Šimanov, On stability of solution of a nonlinear equation of the third order, Akad. Nauk SSSR. Prikl. Mat. Meh. 17 (1953), 369-372.

[13] Y. P. Singh, The asymptotic behavior of solutions of linear third order differential equations, Proc. Amer. Math. Soc. 20 (1969), 309-314.

[14] K. E. Swick, Asymptotic behavior of the solutions of certain third order differential equations, SIAM J. Appl. Math. 19 (1970), 96-102.

[15] L. Wang and M. Q. Wang, On the construction of globally asymptotically stable Lyapunov functions for a type of nonlinear third-order system, Acta Math. Appl. Sinica 6 (1983), no. 3, 309-323.

[16] T. Yoshizawa, Asymptotic behavior of solutions of a system of differential equations, Contributions to Differential Equations 1 (1963), 371-387.

Cemil Tunç: Department of Mathematics, Faculty of Arts and Sciences, Yüzüncü Yıl University, 65080 Van, Turkey

E-mail address: cemtunc@yahoo.com 


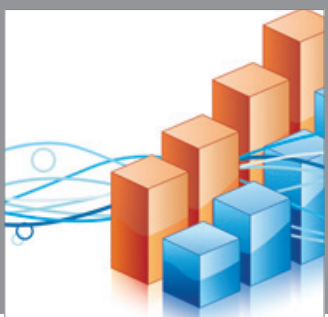

Advances in

Operations Research

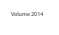

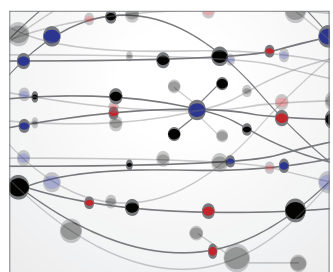

\section{The Scientific} World Journal
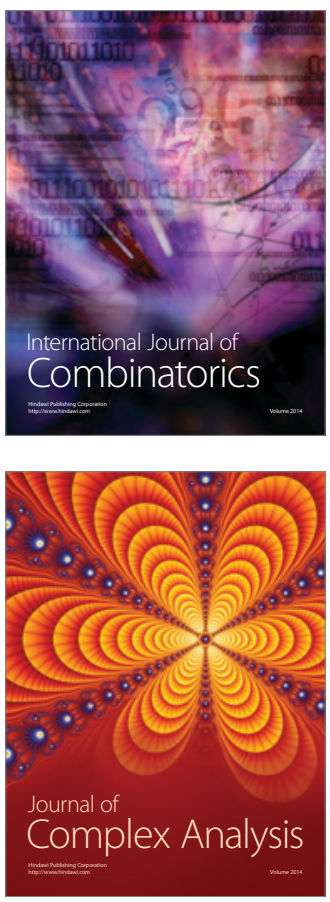

International Journal of

Mathematics and

Mathematical

Sciences
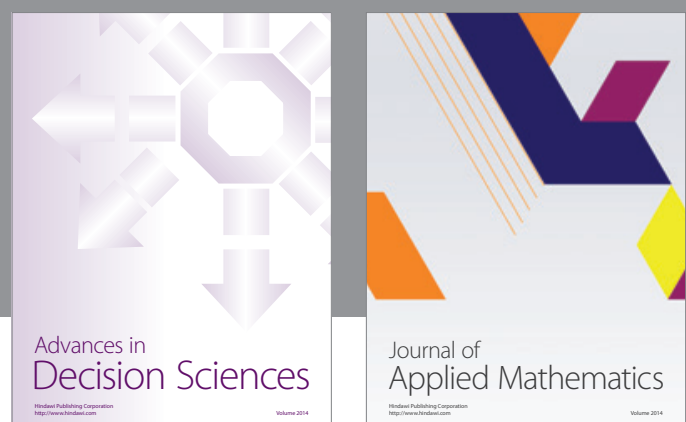

Journal of

Applied Mathematics
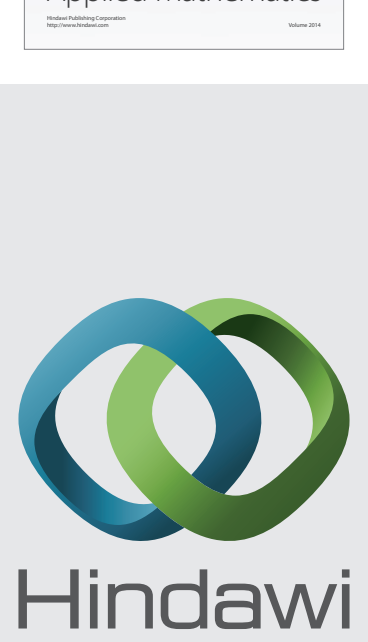

Submit your manuscripts at http://www.hindawi.com
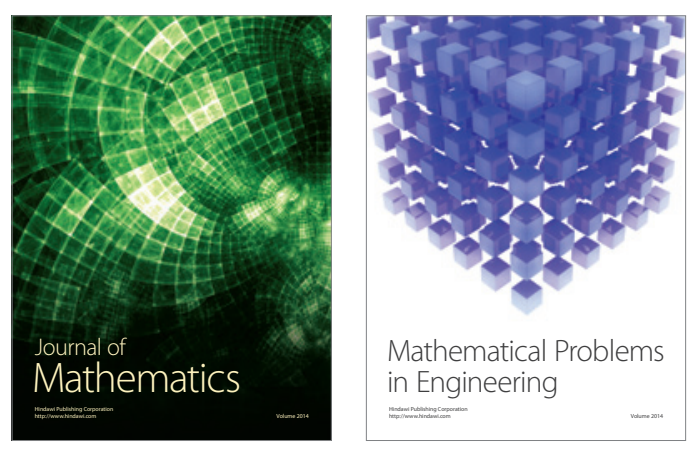

Mathematical Problems in Engineering
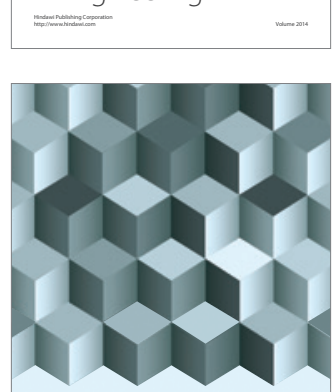

Journal of

Function Spaces
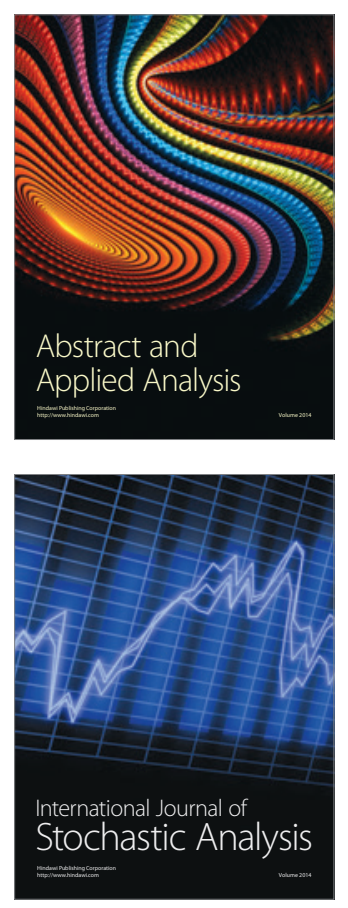

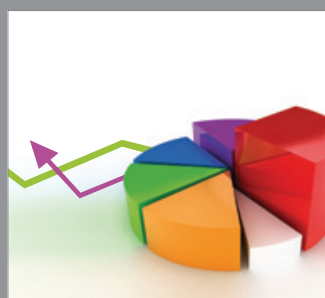

ournal of

Probability and Statistics

Promensencen
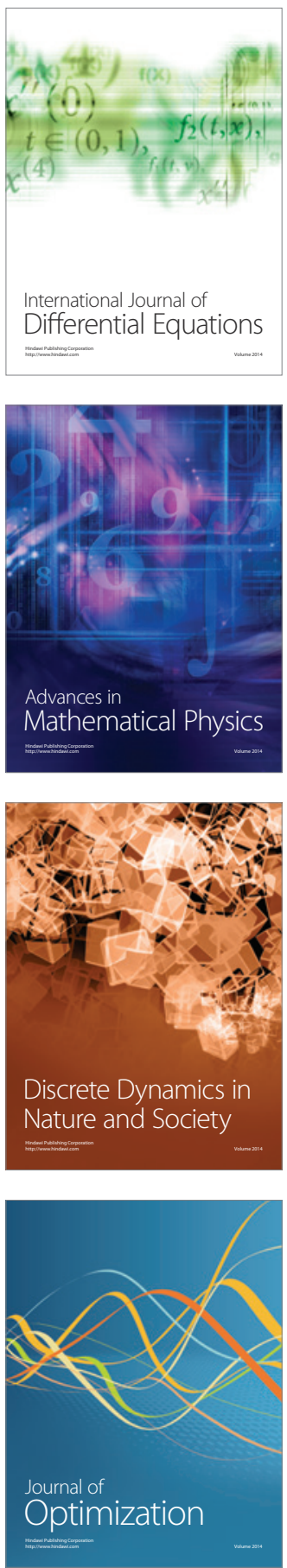